\title{
Analytical solutions forfuzzysystem using power series approach
}

\author{
Sana Abughurra \\ Department of Mathematics, Faculty of Science, Ajloun National University, Ajloun 26816, Jordan
}

\begin{abstract}
The aim of the present paper is present a relatively new analytical method, called residual power series (RPS) method, for solving system of fuzzy initial value problems under strongly generalized differentiability. The technique methodology provides the solution in the form of a rapidly convergent series with easily computable components using symbolic computation software. Several computational experiments are given to show the good performance and potentiality of the proposed procedure. The results reveal that the present simulated method is very effective, straightforward and powerful methodology to solve such fuzzy equations.
\end{abstract}

Keywords: Fuzzy differential equations;Residual power series method;Initial value problems; Strongly generalized differentiability

\section{Introduction}

Fuzzy differential equations (FDEs) are extensively used in modeling of complex phenomena arising in applied mathematics, physics, and engineering, including fuzzy controltheory, quantum optics, atmosphere, measure theory and dynamical systems [1-4]. In many cases, data about these physical phenomena is pervaded under uncertainty, which may arise in the experiment part, data collection, measurement process as well as when determining the initial values. Most of the uncertain practical problems under the differential sense require the solutions of the corresponding FDEs which satisfy the given fuzzy initial conditions; therefore, fuzzy problems should be solved. In general, there exists no method that yields an explicit solution for FDEs due to the complexities of uncertain parameters involving these equations. Therefore, an efficient reliable computer stimulation is required. To deal with this in more realistic situations, FDEs are commonly solved approximately using numerical techniques [5-9].On the other hand, applications for different problems using numerical methods can be found in [10-16].

Series expansions are very important aids in numerical calculations, especially for quick estimates made in hand calculation. Solutions of the FDEs can often be expressed in terms of series expansions. However, the RPS technique is an analytical method for solving different types of ordinary and partial differential equations [17-19]. The methodology is effectiveand easy to construct power series solution for strongly linear and nonlinear systems of FIVPs without linearization, perturbation, or discretization.Different from the classical power series method, the RPS technique does not need to compare the coefficients of the corresponding terms and recursion relations are not required, which computes the coefficients of its power series by a chain of linear equations of $n$-variable, where $n$ is number of equations in the given system. Thetraditional higher order Taylor series method is computationally expensive for large orders and suited for the linear problems, while on the other hand, the proposed method is an alternative procedure for obtaining analytic Taylor series solution of systems of FIVPs. By using residual error concept, we get a series solution, in practice a truncated series solution [20-22]. More specifically, we discuss and provide numerical approximate solutions for system of FIVP of the form

$$
u_{i}^{\prime}(t)=F_{i}\left(t, u_{i}(t)\right), i=1,2, \ldots, n,
$$

subject to the initial conditions

$$
u_{i}\left(t_{0}\right)=\xi_{i}, i=1,2, \ldots, n,
$$

where $t \in\left[t_{0}, t_{0}+a\right], t_{0}, \xi_{i} \in \mathbb{R}, F_{i}:\left[t_{0}, t_{0}+a\right] \times \mathbb{R}^{n} \rightarrow \mathbb{R}$ are nonlinear continuous functions in term of $u_{i}$. The unknown functions $u_{i}(t), i=1,2, \ldots, n$, of independent variable $t$ to be determined.Throughout thispaper, we assume that $F_{i}, i=$ $1,2, \ldots, n$, are analytic functionsand satisfies all the necessary requirements for the existence of a unique solution.

This article is organized as follows. In the next section, we revisit brieflysome necessary definitions and preliminary results from the fuzzy calculus theory including the strongly generalized differentiability. Formulation for solving the system of FIVPs is presented in Section 3.Numerical experiments and simulation results are presented inSection 4 . This article ends in Section 5 with some concluding remarks.

\section{Fuzzy Analysis Theory}

In this section, we present some necessary definitions and notations from fuzzy calculus theory which be used throughout the paper.

Definition 2.1. [23] A fuzzy numberu is a fuzzy subset of the real line with a normal, convex, and upper semicontinuous membership function of bounded support.

Theorem 2.1.[23] Suppose that $\underline{u}:[0,1] \rightarrow \mathbb{R a n d} \bar{u}:[0,1] \rightarrow \mathbb{R}$ satisfy the following conditions: First, $\underline{u}$ is a bounded increasing function and $\bar{u}$ is a bounded decreasing function with $\underline{u}(1) \leq \bar{u}(1)$; second, for each $\alpha \in(0,1], \underline{u}$ and $\bar{u}$ are lefthand continuous functions at $\alpha=r$; third, $\underline{u}$ and $\bar{u}$ are right-hand continuous functions at $r=0$. Then, $u$ : $\mathbb{R} \rightarrow[0,1]$ defined $\operatorname{by} u(s)=\sup \{r: \underline{u}(r) \leq s \leq \bar{u}(r)\}$ is a fuzzy number with parameterization $[\underline{u}(r), \bar{u}(r)]$. Furthermore, if $u: \mathbb{R} \rightarrow[0,1]$ is a fuzzy number with parameterization $[\underline{u}(r), \bar{u}(r)]$,then the functions $\underline{u}$ and $\bar{u}$ satisfy the aforementioned conditions. 
In general, we can represent an arbitrary fuzzy number $u$ by an order pair of functions $(\underline{u}, \bar{u})$ based upon therequirements mentionedin Theorem 2.1. On the other hand, for arbitrary fuzzy numbersu= $(\underline{u}(r), \bar{u}(r)), v=$ $(\underline{v}(r), \bar{v}(r))$ and $\lambda \in \mathbb{R}$,we define the addition and scalar multiplication by $\lambda$ as $[u+v]^{r}=[u]^{r}+[v]^{r}=[\underline{u}(r)+\underline{v}(r), \bar{u}(r)+$ $\nu(r)$ and $\lambda u r=\lambda u r=[\min \{\lambda u(r), \lambda u(r)\}, \max \{\lambda u(r), \lambda u(r)\}]$. Moreover, any two fuzzy numbers $u$ and $v$ are equal, if $u r=v r$ for each $r \in[0,1]$, that is, $\underline{u}(r)=\underline{v}(r)$ and $\bar{u}(r)=\bar{v}(r)$.

The metric structure on $\mathbb{R}_{F}$ is given by the Hausdorff distance $D: \mathbb{R}_{F} \times \mathbb{R}_{F} \rightarrow \mathbb{R}^{+} \cup\{0\}$ such that $D(u, v)=\sup _{0 \leq r \leq 1} \max \{|\underline{u}(r)-\underline{v}(r)|,|\bar{u}(r)-\bar{v}(r)|\}$ for arbitrary fuzzy numbers $u$ and $v$.

Definition 2.2. [24] Let $u, v \in \mathbb{R}_{F}$. If there exists $w \in \mathbb{R}_{F}$ such that $u=v+w$, then wis called the H-difference (Hukuhara difference) of $u$ and $v$, and is denoted by $u \ominus v$.

Definition 2.3. [24] Let $u:[a, b] \rightarrow \mathbb{R}_{F}$ and $t_{0} \in[a, b]$. We say that $x$ is strongly generalized differentiable at $t_{0}$, if there exists an element $u^{\prime}\left(t_{0}\right) \in \mathbb{R}_{F}$ such that either

i) for each $h>0$ sufficiently close to 0 , the H-differences $u\left(t_{0}+h\right) \ominus u\left(t_{0}\right), u\left(t_{0}\right) \ominus u\left(t_{0}-h\right)$ exist and

$$
\lim _{h \rightarrow 0^{+}} \frac{u\left(t_{0}+h\right) \ominus u\left(t_{0}\right)}{h}=\lim _{h \rightarrow 0^{+}} \frac{u\left(t_{0}\right) \ominus u\left(t_{0}-h\right)}{h}=u^{\prime}\left(t_{0}\right),
$$

ii) for each $h>0$ sufficiently close to 0 , the H-differences $u\left(t_{0}\right) \ominus u\left(t_{0}+h\right), u\left(t_{0}-h\right) \ominus u\left(t_{0}\right)$ exist and

$$
\lim _{h \rightarrow 0^{+}} \frac{u\left(t_{0}\right) \ominus u\left(t_{0}+h\right)}{-h}=\lim _{h \rightarrow 0^{+}} \frac{u\left(t_{0}-h\right) \ominus u\left(t_{0}\right)}{-h}=u^{\prime}\left(t_{0}\right) .
$$

Here, the limit is taken in the metric space $\left(\mathbb{R}_{F}, D\right)$ and at the endpoints of $[a, b]$, we consider only one-sided derivatives. If $u$ is differentiable at any point $t \in[a, b]$, then we say that $u$ is differentiable on $[a, b]$. Furthermore, we say that $u$ is(1)-differentiableon $[a, b]$, if $u$ is differentiable in the sense of $(i)$ and its derivative is denoted $D_{1} u$, while $u$ is (2)differentiableon $[a, b]$, if $u$ is differentiable in the sense of $(i i)$ and its derivative is denoted $D_{2} u$.Frequently, we will write simply $u_{1 r}$ and $u_{2 r}$ instead of $\underline{u}(r)$ and $\bar{u}(r)$, respectively, for each $r \in[0,1]$.

Theorem 2.2. [23] Let $u:[a, b] \rightarrow \mathbb{R}_{F}$ and put $[u(t)]^{r}=\left[u_{1 r}(t), u_{2 r}(t)\right]$ for each $r \in[0,1]$.

i) If $u$ is (1)-differentiable, then $u_{1 r}$ and $u_{2 r}$ are differentiable functions and $\left[D_{1} u(t)\right]^{r}=\left[u_{1 r}^{\prime}(t), u_{2 r}^{\prime}(t)\right]$,

ii) If $u$ is (2)-differentiable, then $u_{1 r}$ and $u_{2 r}$ are differentiable functions and $\left[D_{2} u(t)\right]^{r}=\left[u_{2 r}^{\prime}(t), u_{1 r}^{\prime}(t)\right]$.

Definition 2.4. Let $u:[a, b] \rightarrow \mathbb{R}_{F}$. Then, we say that $x$ is continuous at $t_{0} \in[a, b]$ if for every $\varepsilon>0$, there exists $\delta=$ $\delta\left(t_{0}, \varepsilon\right)>0$ such that $D\left(u(t), u\left(t_{0}\right)\right)<\varepsilon$, for eacht $\in[a, b]$, whenever $\left|t-t_{0}\right|<\delta$.

Here, we say that $u$ is continuous on $[a, b]$, if $u$ is continuous at each $t_{0} \in[a, b]$ such that the continuity is one-sided at endpoints of $[a, b]$, that is, $u$ is continuous on $[a, b]$ if and only if $u_{1 r}$ and $u_{2 r}$ arecontinuous on $[a, b]$.

\section{Fuzzy System Initial Value Problems}

In this section, we study the system ofFIVPs under the concept of strongly generalized differentiability in which the fuzzy differential equation is converted into equivalent system of crisp system of IVPs for each type of differentiability. These can be done if the initial value is fuzzy number, the solution is fuzzy function, and consequently the derivative must be considered as fuzzy derivative.

Prior to construct approximate possible fuzzy solutions of fuzzy system (1) and (2), we write the fuzzy functions $u_{i}(t), i=1,2, \ldots, n$, in terms of its $r$-cut representation form to get that $\left[u_{i}(t)\right]^{r}=\left[u_{(i) 1 r}(t), u_{(i) 2 r}(t)\right]$ and $\left[u_{i}\left(t_{0}\right)\right]^{r}=$ $\left[\alpha_{(i) 1 r}, \alpha_{(i) 2 r}\right], i=1,2, \ldots, n$.Thus, by considering the parametric form for both sides of system (1) and (2), one can write

$$
\left[u_{i}(t)\right]^{r}=\left[F_{i}\left(t, u_{k}(t)\right)\right]^{r}, k=1,2, \ldots, n,
$$

subject to the initial conditions

$$
\left[u_{i}\left(t_{0}\right)\right]^{r}=\left[\xi_{i}\right]^{r}, i=1,2, \ldots, n,
$$

where

$$
\left[F_{i}\left(t, u_{k}(t)\right)\right]^{r}=\left[F_{(i) 1 r}\left(t, u_{k}(t)\right), F_{(i) 2 r}\left(t, u_{k}(t)\right)\right]=\left[F_{(i) 1 r}\left(t, u_{(k) 1 r}(t), u_{(k) 2 r}(t)\right), F_{(i) 2 r}\left(t, u_{(k) 1 r}(t), u_{(k) 2 r}(t)\right)\right] .
$$

Definition 3.1. [24] Let $u:[a, b] \rightarrow \mathbb{R}_{F}$ such that $D_{1} u$ and $D_{2} u$ exists. If $u$ with $D_{1} u$ satisfy the fuzzy system (1) and (2), then we say that $u$ is a (1)-solution of FIVPs (1) and (2). Similarly, if $x$ with $D_{2} u$ satisfy the fuzzy system (1) and (2), then we say that $u$ is a (2)-solution of FIVPs (1) and (2).

Let $x$ be a $(n)$-solution, then by utilizing Theorems 2.2, we can thus translate the fuzzy system (1) and (2) into system of crisp DEs, hereafter, called the corresponding $(n)$-system. In some cases, we can't decompose the membership 
function of the fuzzy solution $[u(t)]^{r}$ as a function defined on $\mathbb{R}$ for each $t \in[a, b]$. Then, we can leave the $(n)$-solution in terms of its $r$-cut representation form.

The RPSM consists in expressing the solutions of system (1) and (2) as a power series expansion about the initial point $t=t_{0}$. To achieve our goal, we suppose that these solutions take the form

$$
u_{i}(t)=\sum_{m=0}^{\infty} u_{i, m}(t), i=1,2, \ldots, n,
$$

where $u_{i, m}(t)=a_{i, m}\left(t-t_{0}\right)^{m}$

Notice that in writing out the term corresponding to $m=0$ in PS (5), we have adopted the convention that $\left(t-t_{0}\right)^{0}=1$ even when $t=t_{0}$. If $t=t_{0}$, then all terms of the PS (5)are vanishing for $m \geq 1$ and so.Anyhow, let $u_{i, 0}(t)$ be the initial guesses approximations of $u_{i}(t), i=1,2, \ldots, n$. Then, since theysatisfy the initial conditions $(2)$, we have $u_{i, 0}\left(t_{0}\right)=u_{i}\left(t_{0}\right)=$ $\xi_{i}, i=1,2, \ldots, n$. Thus, after choosing $u_{i, 0}(t)=u_{i}\left(t_{0}\right)$ as initial approximations of $u_{i}(t)$ andcalculating $u_{i, m}(t)$ for $m=$ $1,2, \ldots, k$,thenthe solutions $u_{i}(t)$ of system (1) and (2)can be approximated by the following $k$ th-truncated series

$$
u_{i}^{k}(t)=\sum_{m=0}^{k} a_{i, m}\left(t-t_{0}\right)^{m}, i=1,2, \ldots, n .
$$

To applying the RPSM, we rewrite the system such that

$$
u_{i}^{\prime}(t)-F_{i}\left(t, u_{i}(t)\right)=0, i=1,2, \ldots, n \text {. }
$$

The subsisting of $k$ th-truncated series into system (6) leads to the following definition of $k$ th-residual functions

$$
\begin{aligned}
& \operatorname{Res}_{i}^{k}(t)=\sum_{m=1}^{k} m a_{i, m}\left(t-t_{0}\right)^{m-1} \\
& -F_{i}\left(t, \sum_{m=0}^{k} a_{1, m}\left(t-t_{0}\right)^{m}, \sum_{m=0}^{k} a_{2, m}\left(t-t_{0}\right)^{m}, \ldots, \sum_{m=0}^{k} a_{n, m}\left(t-t_{0}\right)^{m}\right),
\end{aligned}
$$

while the $\infty$ th-residual functions is given $\operatorname{byRes}_{i}^{\infty}(t)=\lim _{k \rightarrow \infty} \operatorname{Res}_{i}^{k}(t), i=1,2, \ldots, n$.

Clear that $\operatorname{Res}_{i}^{\infty}(t)=0$ for each $t \in\left[t_{0}, t_{0}+a\right]$. This show that $\operatorname{Res}_{i}^{\infty}(t)$ are infinitely many times differentiable at $t=t_{0}$. On the other hand, $\frac{d^{n}}{d t^{n}} \operatorname{Res}_{i}^{\infty}\left(t_{0}\right)=\frac{d^{n}}{d t^{n}} \operatorname{Res}_{i}^{k}\left(t_{0}\right)=0$, for each $n=1,2, \ldots, k$.To obtain the values of the coefficients $c_{i, m}$, we need to solve the following algebraic system $\frac{d^{(n-1)}}{d t^{(n-1)}} \operatorname{Res}_{i}^{n}\left(t_{0}\right)=0, n=1,2, \ldots, k$.

Todetermine the first unknown coefficient, $a_{i, 1}$,we defined the 1st-residual function as $\operatorname{Res}_{i}^{1}(t)=\sum_{m=1}^{1} m a_{i, m}\left(t-t_{0}\right)^{m-1}-F_{i}\left(t, \sum_{m=0}^{1} a_{1, m}\left(t-t_{0}\right)^{m}, \ldots, \sum_{m=0}^{1} a_{n, m}\left(t-t_{0}\right)^{m}\right)$. Substituting $t=t_{0}$ into the 1 stresidual function and then using the fact $\operatorname{Res}_{i}^{\infty}\left(t_{0}\right)=\operatorname{Res}_{i}^{1}\left(t_{0}\right)=0$, we have $a_{i, 1}=F_{i}\left(t_{0}, a_{1.0}, a_{2.0}, \ldots, a_{n .0}\right)=f_{i}\left(t_{0}, u_{1}\left(t_{0}\right), u_{2}\left(t_{0}\right), \ldots, u_{n}\left(t_{0}\right)\right), i=1,2, \ldots, n$. Thus, by using the1st-truncated series, the $1 \mathrm{st}-$ approximate solutions can be written as

$$
u_{i}^{1}(t)=u_{i}\left(t_{0}\right)+F_{i}\left(t_{0}, u_{i}\left(t_{0}\right)\right)\left(t-t_{0}\right), i=1,2, \ldots, n .
$$

Forsecond unknown coefficient, $a_{i, 2}$, differentiate both sides of formula(7) with respect to $t$, put $k=2$, and then substitute $t=t_{0}$ to get that

$$
\begin{aligned}
& \frac{d}{d t} \operatorname{Res}_{i}^{2}\left(t_{0}\right)=2 a_{i, 2}-\frac{\partial}{\partial t} F_{i}\left(t_{0}, a_{1.0}, a_{2.0}, \ldots, a_{n .0}\right) \\
& -\sum_{j=1}^{n} a_{j, 1} \frac{\partial}{\partial x_{j}^{2}} F_{i}\left(t_{0}, a_{1,0}, a_{2,0}, \ldots, a_{n, 0}\right), i=1,2, \ldots, n .
\end{aligned}
$$

Similarly, by using the fact $\frac{d}{d t} \operatorname{Res}_{i}^{2}\left(t_{0}\right)=\frac{d}{d t} \operatorname{Res}_{i}^{\infty}\left(t_{0}\right)=0$, we have

$$
\begin{gathered}
a_{i, 2}=\frac{1}{2}\left[\frac{\partial}{\partial t} F_{i}\left(t_{0}, u_{1}\left(t_{0}\right), u_{2}\left(t_{0}\right), \ldots, u_{n}\left(t_{0}\right)\right)+\sum_{j=1}^{n} a_{j, 1} \frac{\partial}{\partial x_{j}^{2}} F_{i}\left(t_{0}, u_{1}\left(t_{0}\right), u_{2}\left(t_{0}\right), \ldots, u_{n}\left(t_{0}\right)\right)\right], i \\
=1,2, \ldots, n .
\end{gathered}
$$

Now, by using the2nd-truncated series, the2nd-approximate solutions can be written as 


$$
\begin{gathered}
u_{i}^{2}(t)=u_{i}\left(t_{0}\right)+F_{i}\left(t_{0}, u_{1}\left(t_{0}\right), u_{2}\left(t_{0}\right), \ldots, u_{n}\left(t_{0}\right)\right)\left(t-t_{0}\right)+\frac{1}{2}\left[\frac{\partial}{\partial t} F_{i}\left(t_{0}, u_{1}\left(t_{0}\right), u_{2}\left(t_{0}\right), \ldots, u_{n}\left(t_{0}\right)\right)\right. \\
\left.+\sum_{j=1}^{n} a_{j, 1} \frac{\partial}{\partial x_{j}^{2}} F_{i}\left(t_{0}, u_{1}\left(t_{0}\right), u_{2}\left(t_{0}\right), \ldots, u_{n}\left(t_{0}\right)\right)\right]\left(t-t_{0}\right)^{2}, i=1,2, \ldots, n .
\end{gathered}
$$

This procedure can be repeated till the arbitrary order coefficients of RPS solutionsare obtained.

Here, we will let $\operatorname{Rem}_{i}^{k}(t)$ denote the difference between $x_{i}(t)$ and its $k$ th Taylor polynomial; that is,

$$
\operatorname{Rem}_{i}^{k}(t)=u_{i}(t)-u_{i}^{k}(t)=\sum_{m=k+1}^{\infty} \frac{u_{i}^{(m)}\left(t_{0}\right)}{m !}\left(t-t_{0}\right)^{m}, i=1,2, \ldots, n,
$$

where the functions $\operatorname{Rem}_{i}^{k}(t)$ are called the $k$ th-remainder for the Taylor series of $u_{i}(t)$.

\section{Numerical Experiments}

To show the simplicity and effectiveness of the proposed algorithm, semi-analytical solutions for a class of both linear and nonlinear inhomogeneous fuzzu IVPs is constructed using the RPS approach. The method is applied in a direct way without using linearization, transformation, or restrictive assumptions. The results reveal that the method is highly accurate, rapidly converge, and convenient to handle a various engineering problems in fuzzy calculus.

Example 4.1Consider the linear fuzzy IVPs in the form

$$
u_{1}^{\prime}(t)-u_{1}(t)-u_{2}(t)=0, t \in[0,1]
$$

$$
u_{2}^{\prime}(t)+u_{1}(t)-u_{2}(t)=0,
$$

subject to the fuzzy initial conditions

$$
u_{1}(0)=\mu_{1}, u_{2}(0)=\mu_{2} \text {, }
$$

where $\mu_{1}(s)=\left\{\begin{array}{ll}s-1, & 1 \leq s \leq 2, \\ 4-s, & 2 \leq s \leq 3,\end{array}\right.$ and $\mu_{2}(s)=\left\{\begin{array}{cc}s, & 0 \leq s \leq 1 \\ 3-s, & 1 \leq s \leq 2\end{array}\right.$

If we put $r=s-1$, and $r=4-s$, then, $\left[\mu_{1}\right]^{r}=[r-1,4-r]$ and $\left[\mu_{2}\right]^{r}=[r, 3-r]$.Using the RPSM, we have that

$$
\begin{aligned}
& u_{1 r}^{\prime}(t)-u_{1 r}(t)-u_{3 r}(t)=0, \\
& u_{2 r}^{\prime}(t)-u_{2 r}(t)-u_{4 r}(t)=0, \\
& u_{3 r}^{\prime}(t)+u_{2 r}(t)-u_{3 r}(t)=0, \\
& u_{4 r}^{\prime}(t)+u_{1 r}(t)-u_{4 r}(t)=0,
\end{aligned}
$$

subject to the fuzzy initial conditions

$$
u_{1 r}(0)=r-1, u_{2 r}(0)=4-r, u_{3 r}(0)=r, u_{4 r}(0)=3-r
$$

Starting with $a_{1,0}=r-1, a_{2,0}=4-r, a_{3,0}=r$, and $a_{4,0}=3-r$, the PSsolutions are

$$
\begin{aligned}
& u_{1 r}(t)=r-1+\sum_{m=1}^{\infty} a_{1, m} t^{m}, \\
& u_{2 r}(t)=4-r+\sum_{m=1}^{\infty} a_{2, m} t^{m}, \\
& u_{3 r}(t)=r+\sum_{m=1}^{\infty} a_{3, m} t^{m}, \\
& u_{4 r}(t)=3-r+\sum_{m=1}^{\infty} a_{4, m} t^{m} .
\end{aligned}
$$

The $k$ th residual functions are given as 


$$
\begin{aligned}
& \operatorname{Res}_{1, r}^{k}(t)=\sum_{m=1}^{k} m a_{1, m} t^{m-1}-\left[\left(r-1+\sum_{m=1}^{k} a_{1, m} t^{m}\right)+\left(r+\sum_{m=1}^{k} a_{3, m} t^{m}\right)\right], \\
& \operatorname{Res}_{2, r}^{k}(t)=\sum_{m=1}^{k} m a_{1, m} t^{m-1}-\left[\left(4-r+\sum_{m=1}^{k} a_{2, m} t^{m}\right)+\left(4-r+\sum_{m=1}^{k} a_{4, m} t^{m}\right)\right], \\
& \operatorname{Res}_{3, r}^{k}(t)=\sum_{m=1}^{k} m a_{3, m} t^{m-1}-\left[-\left(4-r+\sum_{m=1}^{k} a_{2, m} t^{m}\right)+\left(r+\sum_{m=1}^{k} a_{3, m} t^{m}\right)\right], \\
& \operatorname{Res}_{4, r}^{k}(t)=\sum_{m=1}^{k} m a_{4, m} t^{m-1}-\left[-\left(1-r+\sum_{m=1}^{k} a_{1, m} t^{m}\right)+\left(4-r+\sum_{m=1}^{k} a_{4, m} t^{m}\right)\right] .
\end{aligned}
$$

Using $N=10$, the RPS solutions are given by

$$
\begin{aligned}
& u_{1 r}(t)=(r-1)(\left.+2 t+2^{2} \frac{t^{2}}{2 !}+2^{3} \frac{t^{3}}{3 !}+2^{4} \frac{t^{4}}{4 !}+2^{5} \frac{t^{5}}{5 !}\right) \\
&+\left(1+t+\frac{t^{2}}{2 !}+\frac{t^{3}}{3 !}+\frac{t^{4}}{4 !}+\frac{t^{5}}{5 !}\right)\left(\mathrm{t}-\frac{t^{3}}{3 !}+\frac{t^{5}}{5 !}\right) \\
&+2\left(1+t+\frac{t^{2}}{2 !}+\frac{t^{3}}{3 !}+\frac{t^{4}}{4 !}+\frac{t^{5}}{5 !}\right)\left(1-\frac{t^{2}}{2 !}+\frac{t^{4}}{4 !}\right), \\
& u_{2 r}(t)=(1-r)(\left.1+2 t+2^{2} \frac{t^{2}}{2 !}+2^{3} \frac{t^{3}}{3 !}+2^{4} \frac{t^{4}}{4 !}+2^{5} \frac{t^{5}}{5 !}\right) \\
&+\left(1+t+\frac{t^{2}}{2 !}+\frac{t^{3}}{3 !}+\frac{t^{4}}{4 !}+\frac{t^{5}}{5 !}\right)\left(t-\frac{t^{3}}{3 !}+\frac{t^{5}}{5 !}\right) \\
&+2\left(1+t+\frac{t^{2}}{2 !}+\frac{t^{3}}{3 !}+\frac{t^{4}}{4 !}+\frac{t^{5}}{5 !}\right)\left(1-\frac{t^{2}}{2 !}+\frac{t^{4}}{4 !}\right), \\
& u_{3 r}(t)=(r-1)\left(1+2 t+2^{2} \frac{t^{2}}{2 !}+2^{3} \frac{t^{3}}{3 !}+2^{4} \frac{t^{4}}{4 !}+2^{5} \frac{t^{5}}{5 !}\right) \\
&+\left(1+t+\frac{t^{2}}{2 !}+\frac{t^{3}}{3 !}+\frac{t^{4}}{4 !}+\frac{t^{5}}{5 !}\right)\left(1-\frac{t^{2}}{2 !}+\frac{t^{4}}{4 !}\right) \\
&-2\left(1+t+\frac{t^{2}}{2 !}+\frac{t^{3}}{3 !}+\frac{t^{4}}{4 !}+\frac{t^{5}}{5 !}\right)\left(t-\frac{t^{3}}{3 !}+\frac{t^{5}}{5 !}\right), \\
&+\left(1+2 t+2^{2} \frac{t^{2}}{2 !}+2^{3} \frac{t^{3}}{3 !}+2^{4} \frac{t^{4}}{4 !}+2^{5} \frac{t^{5}}{5 !}\right) \\
&+\left(1+t+\frac{t^{2}}{2 !}+\frac{t^{3}}{3 !}+\frac{t^{4}}{4 !}+\frac{t^{5}}{5 !}\right)\left(1-\frac{t^{2}}{2 !}+\frac{t^{4}}{4 !}\right) \\
&-2\left(1+t+\frac{t^{2}}{2 !}+\frac{t^{3}}{3 !}+\frac{t^{4}}{4 !}+\frac{t^{5}}{5 !}\right)\left(t-\frac{t^{3}}{3 !}+\frac{t^{5}}{5 !}\right) .
\end{aligned}
$$

Example 4.2 Consider the nonlinear system of fuzzy IVPs

$$
\begin{aligned}
& u_{1}^{\prime}(t)-e^{u_{2}(t)}=\mu, t \in[0,1], \\
& u_{2}^{\prime}(t)-\left(u_{1}(t)\right)^{3}=0,
\end{aligned}
$$

subject to the fuzzy initial conditions

$$
u_{1}(0)=0, u_{2}(0)=\eta \text {, }
$$

where $\mu(s)=\max _{s \in \mathbb{R}}\left(0,1-(4 s)^{\frac{2}{3}}\right)$ and $\eta(s)=\max _{s \in \mathbb{R}}\left(0.1-(5 s)^{2}\right)$.

Now, weput $\quad u_{1 r}(t)=\left[u_{1 r}(t), u_{2 r}(t)\right] \quad$ and $u_{2 r}(t)=\left[u_{3 r}(t), u_{4 r}(t)\right], \quad$ then, $\quad$ we have that $u_{1 r}^{3}(t)=\left[u_{1 r}^{3}(t), u_{2 r}^{3}(t)\right]$ and $\left(e^{u_{2}(t)}\right)_{r}=\left[e^{u_{3 r}(t)}, e^{u_{4 r}(t)}\right]$.Thus, if $\quad r=1-(4 s)^{\frac{2}{3}}$, thens $=-\frac{1}{4}(1-r)^{\frac{3}{2}}$ or $s=\frac{1}{4}(1-r)^{\frac{3}{2}}$; Therefore, $\mu_{r}=\left[-\frac{1}{4} \sqrt{(1-r)^{3}}, \frac{1}{4} \sqrt{(1-r)^{3}}\right]$;and $\eta_{r}=\left[-\frac{1}{5} \sqrt{1-r}, \frac{1}{5} \sqrt{1-r}\right]$.

Starting with the initial data $a_{1,0}=-\frac{1}{4} \sqrt{(1-r)^{3}}, a_{2,0}=\frac{1}{4} \sqrt{(1-r)^{3}}, a_{3,0}=\left[-\frac{1}{5} \sqrt{1-r}\right.$, and $a_{4,0}=\frac{1}{5} \sqrt{1-r}$. Then, regarding the RPSM, the 10th-truncated PSsolutions have to be in the form 


$$
\begin{aligned}
& u_{1 r}^{10}(t)=\left[e^{-\frac{1}{5} \sqrt{1-r}}-\frac{1}{4} \sqrt{(1-r)^{3}}\right] t+\left[\frac{1}{20} e^{-\frac{1}{5} \sqrt{1-r}}\left(e^{\left(-\frac{1}{5} \sqrt{1-r}\right)}-\frac{1}{4} \sqrt{(1-r)^{3}}\right)^{3}\right] t^{5} \\
& +\left[\frac{1}{288} e^{-\frac{1}{5} \sqrt{1-r}}\left(e^{-\frac{1}{5} \sqrt{1-r}}-\frac{1}{4} \sqrt{(1-r)^{3}}\right)^{6}\right. \\
& \left.+\frac{1}{480} e^{-\frac{2}{5} \sqrt{1-r}}\left(e^{-\frac{1}{5} \sqrt{1-r}}-\frac{1}{4} \sqrt{(1-r)^{3}}\right)^{5}\right] t^{9} \\
& u_{2 r}^{10}(t)=\left[e^{-\frac{1}{5} \sqrt{1-r}}+\frac{1}{4} \sqrt{(1-r)^{3}}\right] t+\left[\frac{1}{20} e^{-\frac{1}{5} \sqrt{1-r}}\left(e^{\left(-\frac{1}{5} \sqrt{1-r}\right)}+\frac{1}{4} \sqrt{(1-r)^{3}}\right)^{3}\right] t^{5} \\
& +\left[\frac{1}{288} e^{-\frac{1}{5} \sqrt{1-r}}\left(e^{-\frac{1}{5} \sqrt{1-r}}-\frac{1}{4} \sqrt{(1-r)^{3}}\right)^{6}\right. \\
& \left.+\frac{1}{480} e^{-\frac{2}{5} \sqrt{1-r}}\left(e^{-\frac{1}{5} \sqrt{1-r}}-\frac{1}{4} \sqrt{(1-r)^{3}}\right)^{5}\right] t^{9} \\
& u_{3 r}^{10}(t)=-\frac{1}{5} \sqrt{1-r}+\frac{1}{4}\left[e^{-\frac{1}{5} \sqrt{1-r}}-\frac{1}{4} \sqrt{(1-r)^{3}}\right]^{3} t^{4} \\
& +\frac{3}{160}\left[e^{-\frac{1}{5} \sqrt{1-r}}\left(e^{\left(-\frac{1}{5} \sqrt{1-r}\right)}-\frac{1}{4} \sqrt{(1-r)^{3}}\right)^{5}\right] t^{8}, \\
& u_{4 r}^{10}(t)=\frac{1}{5} \sqrt{1-r}+\frac{1}{4}\left[e^{-\frac{1}{5} \sqrt{1-r}}+\frac{1}{4} \sqrt{(1-r)^{3}}\right]^{3} t^{4} \\
& +\frac{3}{160}\left[e^{-\frac{1}{5} \sqrt{1-r}}\left(e^{\left(-\frac{1}{5} \sqrt{1-r}\right)}+\frac{1}{4} \sqrt{(1-r)^{3}}\right)^{5}\right] t^{8} \text {. }
\end{aligned}
$$

\section{Conclusion}

In this paper, a new analytic-numeric method, so-called RPSM, is proposed and applied to handle the both linear and nonlinear fuzzy system of IVPs. Numerical results reveal the complete reliability and efficiency of the proposed method with a great potential in scientific applications. It may be concluded that the RPSM is very powerful, straightforward, and promising technique in finding analytic approximate solution for wide classes of fuzzy IVPs.

\section{References}

[1] D. Li, M. Chen, X. Xue, Two-point boundary value problems of uncertain dynamical systems, Fuzzy Sets and Systems, 179 (2011), 50-61.

http://dx.doi.org/10.1016/j.fss.2011.05.012

[2] R. Goetschel, W. Voxman, Elementary fuzzy calculus, Fuzzy Sets and Systems,18 (1986), $31-43$. http://dx.doi.org/10.1016/0165-0114(86)90026-6

[3] D.H. Ackley, G.E. Hinton and T.J. Sejnowski, A learning algorithm for Boltzmann machine, Cognitive Science, 9 (1985), 147-169. http://dx.doi.org/10.1016/s0364-0213(85)80012-4

[4] A. Khastan, F. Bahrami, K. Ivaz, New Results on multiple solutions for Nth-order fuzzy differential equations under generalized differentiability, Boundary Value Problems, 2009 (2009), Article ID 395714, 1-13.

http://dx.doi.org/10.1155/2009/395714

[5] O. Abu Arqub, M. Al-Smadi, S. Momani and T. Hayat, Numerical Solutions of Fuzzy Differential Equations using Reproducing Kernel Hilbert Space Method, Soft Computing, 2015 (2015), 1-20. http://dx.doi.org/10.1007/s00500015-1707-4

[6] O. Abu Arqub, Adaptation of reproducing kernel algorithm for solving fuzzy Fredholm-Volterra integrodifferential equations, Neural Computing and Applications, 2015 (2015), 1-20. http://dx.doi.org/10.1007/s00521-015-2110-x

[7] G. Gumah, K. Moaddy, M. AL-Smadi and I. Hashim, Solutions of Uncertain Volterra Integral Equations by Fitted Reproducing Kernel Hilbert Space Method, Journal of Function Spaces, 2016 (2016), Article ID 2920463, 1-11. http://dx.doi.org/10.1155/2016/2920463

[8] O. Abu Arqub, M. Al-Smadi, S. Momani and T. Hayat, Application of reproducing kernel algorithm for solving second-order, two-point fuzzy boundary value problems, Soft Computing, 2016 (2016), 1-16. doi:10.1007/s00500016-2262-3 
[9] R. Saadeh, M. Al-Smadi, G. Gumah, H. Khalil and R.A. Khan, Numerical Investigation for Solving Two-Point Fuzzy Boundary Value Problems by Reproducing Kernel Approach, Applied Mathematics and Information Sciences, 10 (2016), no. 6, 1-13.

[10] I. Komashynska and M. Al-Smadi, Iterative reproducing kernel method for solving second-order integrodifferentia equations of Fredholm Type, Journal of Applied Mathematics, 2014 (2014), Article ID 459509, 1-11. http://dx.doi.org/10.1155/2014/459509

[11] M. Al-Smadi, O. Abu Arqub and A. El-Ajou, A numerical iterative method for solving systems of first-order periodic boundary value problems, Journal of Applied Mathematics, 2014 (2014), Article ID 135465, 1-10. http://dx.doi.org/10.1155/2014/135465

[12] O. Abu Arqub, M. Al-Smadi and S. Momani, Application of reproducing kernel method for solving nonlinear Fredholm-Volterra integrodifferential equations, Abstract and Applied Analysis, 2012 (2012), Article ID 839836, 1-16. http://dx.doi.org/10.1155/2012/839836

[13] O. Abu Arqub and M. Al-Smadi, Numerical algorithm for solving two-point, second-order periodic boundary value problems for mixed integro-differential equations, Applied Mathematical and Computation, 243 (2014), 911-922. http://dx.doi.org/10.1016/j.amc.2014.06.063

[14] M. Al-Smadi, O. Abu Arqub and S. Momani, A Computational Method for Two-Point Boundary Value Problems of Fourth-Order Mixed Integrodifferential Equations, Mathematical Problems in Engineering, 2013 (2013), Article ID 832074, 1-10. http://dx.doi.org/10.1155/2013/832074

[15] O. Abu Arqub, M. Al-Smadi and N. Shawagfeh, Solving Fredholm integro-differential equations using reproducing kernel Hilbert space method, Applied Mathematics and Computation, 219 (2013), 8938-8948. http://dx.doi.org/10.1016/j.amc.2013.03.006

[16] M. Al-Smadi, O. Abu Arqub, N. Shawagfeh and S. Momani, Numerical investigations for systems of secondorder periodic boundary value problems using reproducing kernel method, Applied Mathematical and Computation, 291 (2016), 137-148. http://dx.doi.org/10.1016/j.amc.2016.06.002

[17] I. Komashynska, M. Al-Smadi, O. Abu Arqub, S. Momani, An efficient analytical method for solving singular initial value problems of nonlinear systems, Applied Mathematics \& Information Sciences, 10 (2016), 647-656. http://dx.doi.org/10.18576/amis/100224

[18] K. Moaddy, M. AL-Smadi and I. Hashim, A Novel Representation of the Exact Solution for Differential Algebraic Equations System Using Residual Power-Series Method, Discrete Dynamics in Nature and Society, 2015 (2015),Article ID 205207, 1-12. http://dx.doi.org/10.1155/2015/205207

[19] I. Komashynska, M. Al-Smadi, A. Ateiwi and S. Al-Obaidy, Approximate analytical solution by residual power series method for system of Fredholm integral equations, Applied Mathematics and Information Sciences, 10 (2016), 975985. http://dx.doi.org/10.18576/amis/100315

[20] A. El-Ajou, O. Abu Arqub and M. Al-Smadi, A general form of the generalized Taylor's formula with some applications, Applied Mathematical and Computation, $256 \quad$ (2015), 851-859. http://dx.doi.org/10.1016/j.amc.2015.01.034

[21] M. Al-Smadi, Solving initial value problems by residual power series method, Theoretical Mathematics and Applications, 3 (2013), 199-210.

[22] O. Abu Arqub, Series solution of fuzzy differential equations under strongly generalized differentiability, Journal of Advanced Research in Applied Mathematics, 5 (2013), 31-52.

[23] O. Kaleva, Fuzzy differential equations, Fuzzy Sets and Systems,24 (1987), 301-317. http://dx.doi.org/10.1016/0165-0114(87)90029-7

[24] M.L. Puri, Fuzzy random variables, Journal of Mathematical Analysis and Applications, 114 (1986), $409-422$. http://dx.doi.org/10.1016/0022-247x(86)90093-4 